\title{
Similarity, global matching, and judgments of frequency
}

\author{
DOUGLAS L. HINTZMAN \\ University of Oregon, Eugene, Oregon
}

\begin{abstract}
In the test-pair similarity effect, forced-choice recognition is more accurate for similar test pairs, such as leopard-cheetah, than it is for unrelated test pairs, such as leopard-turnip. According to global matching models, this occurs because the retrieved familiarities of similar items are correlated. In the Minerva 2 model, global matching underlies frequency judgments as well as recognition memory. One implication of this model is that judged frequencies of similar items should be correlated. Another implication is that judgments of summed frequency for pairs of words (how many presentations were there of word ${ }_{1}$ and word combined?) should have higher variance when word $_{1}$ and word are similar $^{2}$ than when they are unrelated. These predictions were tested and confirmed in two experiments. A review of these and other results suggests that theories of recognition memory should also be applicable to frequency-judgment tasks.
\end{abstract}

In global-matching models of recognition memory (Eich, 1982; Gillund \& Shiffrin, 1984; Hintzman, 1988; Humphreys, Bain, \& Pike, 1989; Murdock, 1982), a retrieval cue does not isolate a specific memory trace, but rather activates many traces simultaneously, each to a degree depending on its similarity to the cue. The retrieved information is a scalar value (variously referred to as strength, familiarity, similarity, or echo intensity) that represents the sum of memory activation produced by the cue. Old-new recognition judgments are made relative to a criterion on the summed activation scale, and forced-choice recognition judgments are made by choosing the test alternative whose summed activation is the highest. Clark and Gronlund (1996) discuss some of the successes and failures of these models.

One success of global-matching models has been in explaining the test-pair similarity effect-a phenomenon of forced-choice recognition. Consider two unrelated items, $\mathrm{A}$ and $\mathrm{B}$, both of which are old with respect to the study list, and two new items, $\mathrm{A}^{\prime}$ ( similar to A) and $B^{\prime}$ (similar to B). Two-alternative forced-choice performance is better on similar pairs such as $A$ versus $A^{\prime}$ than it is on dissimilar pairs such as A versus $B^{\prime}$. In the first case, the lure is related to the old test item with which it is paired, and in the second case, it is related to an old item that is not part of the same test pair. The test-pair similarity effect has been found in experiments manipulating the orthographic similarity of words (Hall, 1979), the semantic similarity of words (Hintzman, 1988), and the visual similarity of photographs (Tulving, 1981). An exception is that the opposite effect-a similar-pair disadvantage-occurs in associative recognition (Clark, Hori, \& Callan, 1993).

Thanks are due Michael Anderson for commenting on an early draft of this article. Correspondence should be addressed to D. L. Hintzman, Department of Psychology, University of Oregon, Eugene, OR 97403 (e-mail: hintzman@oregon.uoregon.edu).
The test-pair similarity prediction derives from a fundamental property of global matching models, which is that correlated inputs or cues produce correlated outputs. Because cues $\mathrm{A}$ and $\mathrm{A}^{\prime}$ are similar, they tend to activate the same subset of memory traces, prominently including the trace of A itself. Thus, if A produces summed activation that is relatively high for an old test item, $\mathrm{A}^{\prime}$ will tend to cause activation that is relatively high for a new item. If A produces activation that is low for an old item, $\mathrm{A}^{\prime}$ is likely to produce activation that is low for a new item. No such correlation is predicted for the unrelated test pair, A versus $\mathrm{B}^{\prime}$.

The choice between an old test item and a new test item is made by selecting the item with the higher summed activation; thus the probability of an error is $P[(X-Y)<$ $0]$, where $X$ is activation caused by the old item, and $Y$ is activation caused by the new item. In the simplest model, the old- and new-item means $\left(\mu_{X}\right.$ and $\left.\mu_{Y}\right)$ and variances $\left(\sigma_{X}^{2}\right.$ and $\sigma_{Y}^{2}$ ) are assumed to be unaffected by the type of test pairing. Thus, $P[(X-Y)<0]$ increases as a direct function of the variance of the differences, $X-Y$ :

$$
\sigma_{X-Y}^{2}=\sigma_{X}^{2}+\sigma_{Y}^{2}-2 \rho_{X Y} \sigma_{X} \sigma_{Y},
$$

where $\rho_{X Y}$ is the Pearson correlation relating $X$ and $Y$. When the old item is A and the new item is $\mathrm{B}^{\prime}, \rho$ is zero and the error rate is at its maximum. When the old item is $\mathrm{A}$ and the new item is $\mathrm{A}^{\prime}, \rho$ is positive and the error rate is reduced. To see intuitively why this happens, consider the extreme (and unattainable) case where $\rho=1.0$. A perfect correlation implies that $X$ and $Y$ fall at exactly corresponding locations in their respective distributions - that is, $z_{X}=z_{Y}$. Thus (as long as $\mu_{X}>\mu_{Y}$ ), $X$ must be greater than $Y$, and $P[(X-Y)<0]=0$.

As applied to forced-choice recognition, Equation 1 is an example of Thurstone's Law of comparative judgment (Thurstone, 1927). Further discussion of this explanation of the test-pair similarity effect can be found in 
Clark and Gronlund (1996, p. 48) and in Hintzman (1988, pp. 538-539).

Global matching models have been used to explain performance in a variety of tasks, in addition to recognition memory. One of these models, Minerva 2 (Hintzman, 1988) has been applied extensively to judgments of presentation frequency (JOFs). A point that is essential to the present study is that in the Minerva 2 model, recognition and JOFs are both based on summed activation. Indeed, because this model assumes that repetition produces multiple memory traces, increasing an item's presentation frequency directly increases the activation the item triggers when used as a test cue. Responding new versus old in recognition is the same as responding JOF $=0$ versus JOF $>0$ in frequency judgments, and forced-choice recognition is the same as frequency discrimination when one alternative has a frequency of zero. To date, none of the other global matching models has been applied to JOFs (but see Gillund \& Shiffrin, 1984, p. 48; Murdock, 1999).

The present study capitalizes on the fact that correlated inputs produce correlated outputs, to test Minerva 2's global-matching account of memory for frequency. Experiment 1 examined correlations between JOFs to similar words, such as A and $\mathrm{A}^{\prime}$. (In this case, $\mathrm{A}$ and $\mathrm{A}^{\prime}$ both may have occurred, at different locations in the study list.) If apparent frequency is based on global matching, then the JOFs should be positively correlated.

Equation 1 has a companion equation, which provided the basis for Experiment 2. When two variables are summed, the variance of $\mathrm{X}+\mathrm{Y}$ is given by

$$
\sigma_{X+Y}^{2}=\sigma_{X}^{2}+\sigma_{Y}^{2}+2 \rho_{X Y} \sigma_{X} \sigma_{Y} .
$$

In contrast to the variance of the difference (Equation 1) the variance of the sum increases with the correlation between $X$ and $Y$. In Experiment 2, subjects saw pairs of test words, for which they gave judgments of summed frequency (JOSFs), intended to reflect the combined frequencies of both words of a test pair. If such judgments are based on global matching, then according to Equation 2, JOSFs for related pairs such as A and $\mathrm{A}^{\prime}$ should be more variable than those for unrelated pairs such as A and $\mathrm{B}^{\prime}$.

\section{EXPERIMENT 1}

In this experiment, subjects studied a list of single words that included semantically related pairs. One member of each pair (A or B) occurred in the first half of the list, and the other member $\left(\mathrm{A}^{\prime}\right.$ or $\left.\mathrm{B}^{\prime}\right)$ occurred in the second half of the list. Presentation frequencies ranged from 0 to 4 and were the same for both members of a given pair. ${ }^{1}$ At test, subjects gave JOFs to individual test words. The analysis of primary interest examined the correlation between JOFs for related test items within each frequency condition (i.e., with objective frequency controlled).

\section{Method}

Subjects. Twenty-three University of Oregon students participated for course credit. They were tested in groups of 2-6 subjects each.
Materials. Fifty pairs of English nouns were selected on the basis of semantic similarity. The aim was to maximize intrapair similarity and minimize interpair similarity. Examples are lawyer-attorney, butter-margarine, ape-gorilla, crayfish-lobster, and pecan-almond. Ten pairs were assigned at random to each of five different presentation frequencies: $0,1,2,3$, and 4 . One member of each pair was assigned at random to the first half of the presentation list and the other member to the second half of the list. The appropriate number of copies of each word was generated, and the first-half and secondhalf words were arranged in separate orders. The orders were random, with the constraint that no word could occur twice in succession. Fifteen filler words were then added to the list -10 at the beginning, occurring one to three times each and 5 at the end, occurring one time each. Altogether, the fillers added 15 presentations to the beginning of the list and 5 at the end, for a total list length of 220 items. In contrast to the experimental words, semantic pair-mates of the filler words were never presented.

Five versions of the study list were constructed by rotating experimental words systematically through the five presentation frequencies. New first-half and second-half orders were also generated. In all five study lists, however, the same filler words appeared in the same positions.

A single 100-word test list was constructed in which the experimental words occurred one time each. The first-half and second-half words appeared in the corresponding halves of the test list. Within list halves, the order of test words was random. Subjects responded to test items on a form that displayed four columns of 25 rows each. Each row listed the item number $(1-100)$, followed by the digits 0-5. JOFs were made by circling a digit for each test item.

Procedure. Each of the five groups of subjects saw a different study list. The 2-6 subjects in each group were seated where they had a clear view of the screen of a Macintosh SE computer. They were instructed orally that a list of 220 words would be shown on the screen, that some of the words would be repeated, and that they were to study the words for a memory test, the nature of which could not be revealed until later. The words were then presented in the center of the computer screen in uppercase 48-point Helvetica font at a rate of one word every $1.5 \mathrm{sec}$.

Following the study list, test forms were distributed and instructions for the JOF test were given. Subjects were told that 100 words would be presented and that they were to indicate the presentation frequency of each word by circling one of the digits, $0-5$ on the test form. They were urged to circle one number for each test word, in its proper order, without skipping any. The test words were then shown at a 6 -sec pace- $5 \mathrm{sec}$ per word with a 1 -sec interstimulus interval (ISI). Presentation numbers $(1-100)$ appeared on the left of the screen, and words appeared on the right in the same font that was used at study. To keep subjects on pace, a beep sounded in the first $0.5 \mathrm{sec}$ of each ISI.

\section{Results}

One subject skipped a line on the test form. This subject's mean JOFs for frequencies $0-4$ also correlated poorly with objective frequency $(r=.39$ compared with a mean of .96 for the other subjects). This person's data were therefore dropped from further analysis, leaving $N=22$ subjects.

Mean JOFs for first-half and second-half words are shown in the top panel of Figure 1, and mean $S D$ s (averaged across subjects) are shown in the bottom panel of Figure 1. First-half JOFs tracked frequency somewhat better than second-half JOFs did. Data were evaluated with orthogonal contrasts, using the $t$-test method outlined by Rosenthal and Rosnow (1985, pp. 56-62). The same three contrasts were done on mean JOFs and mean $S D$ s. 


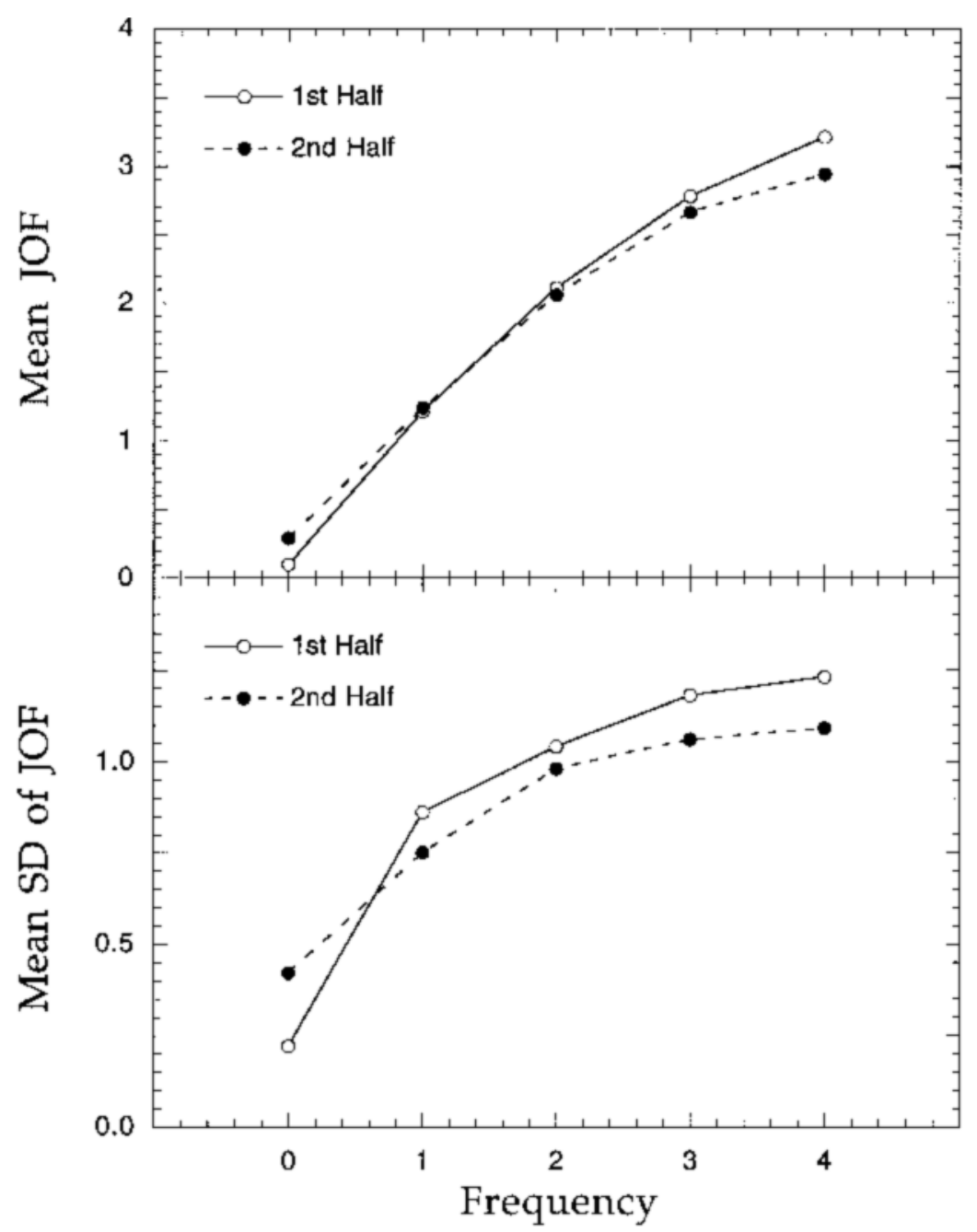

Figure 1. Mean judgment of frequency (JOF) data from Experiment 1.

Because the pattern of significance and nonsignificance was the same on both measures, the tests are reported together (means first and $S D$ s second). The linear trend on frequency was highly significant $[t(21)=22.29$ and 9.50]. First-half versus second-half was unreliable as a main effect $[t(21)=0.65$ and 1.07], but its interaction with frequency was significant $[t(21)=3.00$ and 2.43 , $p=.007$ and .024].

The experiment is not analytic regarding the cause of the interactions, because half-list during study was confounded with half-list during test. Encoding effects (e.g., attention) and test effects (e.g., proactive interference, growing carelessness in responding) may both contribute to the interaction. All second-half tests $\left(\mathrm{A}^{\prime}\right)$ were of course also preceded by a first-half test on the related word (A), but this did not uniformly raise JOFs, as one might have expected. Instead, JOFs tended to regress toward the overall average in the second half of the list; that is, means and $S D$ s became more uniform across frequencies. A sim- ilar regression in mean JOFs, over a 24 -h retention interval, can be seen in the data of Leicht (1968).

The use of a closed judgment scale with a maximum JOF of 5 undoubtedly contributed to the forms of the functions in Figure 1. An open-ended scale probably would have produced higher means and $S D$ s, especially at the larger frequencies. An advantage of the closed scale is that it avoids occasional erratic JOFs such as 10 or 50, which can contribute disproportionately to measurement error.

The focus of interest here is on JOF correlations. To estimate correlations, each subject's data were first sorted by presentation frequency. Within a frequency, JOFs made to first-half words (A) were juxtaposed with JOFs to their second-half pair mates $\left(\mathrm{A}^{\prime}\right)$, and a different Pearson $r$ was computed for each frequency level (0-4) for each subject. A potential problem in interpreting such $r$ values is that they do not distinguish between covariance attributable to episodic encoding and covariance attributable to extraneous properties of the words. Paired 
words are categorically alike, and they may also be similar in word frequency, spelling, length, and other properties that could potentially influence JOFs. To control for such factors, a set of between-subjects $r$ values was also computed, relating the first-half JOFs of each subject with the second-half JOFs of a different subject who had studied the same list. To the extent that JOFs are influenced by nonepisodic properties of the word pairs, the between-subjects $r$ values should tend to be positive. ${ }^{2}$

The large majority of JOFs were correct at a frequency of 0 . For this reason, in 15 out of 22 of the subjects' data, either the first-half or second-half judgments displayed no variance, making it impossible to compute $r$. Zerofrequency correlations will therefore not be considered further. Figure 2 shows the mean Pearson $r$ s for frequencies 1-4, computed both within subjects and between subjects. Within-subjects correlations were positive-as predicted-especially at frequencies of 3 and 4 , whereas between-subjects correlations were near zero. Averaged over frequencies, the mean within-subjects $r(0.223)$ was significantly different from zero $[t(21)=7.09]$, but the mean between-subjects $r(.047)$ was not $[t(21)=1.76$, $p=.093]$.

Analyses again made use of orthogonal planned contrasts. Within-subjects $r$ s were reliably greater than between-subjects $r$ s $[t(21)=4.73, p<.0001]$. However, both the linear trend over the frequencies 1-4 and the interaction of this trend with within- versus betweensubjects fell short of significance $[t(21)=1.11$ and 1.70$]$. The primary finding - the statistically significant withinsubjects correlation-is as predicted by the hypothesis that JOFs are based on global matching.

\section{EXPERIMENT 2}

In this experiment, the study conditions were exactly the same as in Experiment 1. On each test trial, however, subjects saw a pair of related words (A and $\mathrm{A}^{\prime}$ ) or a pair of unrelated words (A and $\left.\mathrm{B}^{\prime}\right)$. They responded with a JOSF that was meant to include both members of the test pair. The prediction of primary interest, in accordance with Equation 2, was that JOSF variability would be greater for related pairs than for unrelated pairs.

An additional question arising from this design relates to the assumption mentioned earlier that $\mu_{X}$ and $\mu_{Y}$ are unaffected by test-pair similarity. To judge combined frequency, assume that the subject attends to one member of the test pair and then quickly switches to the other, retrieving a strength or summed activation for each. To the extent that episodic retrieval is affected by semantic priming, this could produce greater total activation and hence higher JOSFs for related pairs such as $A$ and $A^{\prime}$ than for unrelated pairs such as $A$ and $\mathrm{B}^{\prime}$. In the context of global activation models, a compound cue mechanism could be invoked to explain such an outcome (Ratcliff \& McKoon, 1988).

\section{Method}

Subjects. A total of 45 subjects were recruited in the same way as in Experiment 1 . They were tested in 10 groups of 3-5 subjects each.

Materials and Procedure. Prior to the test instructions, the procedure was identical to that in Experiment 1. The same five study lists were used.

The test instructions introduced the JOSF task, which required subjects to respond to each test pair with a numerical judgment of the combined frequencies of both words. The test list consisted of

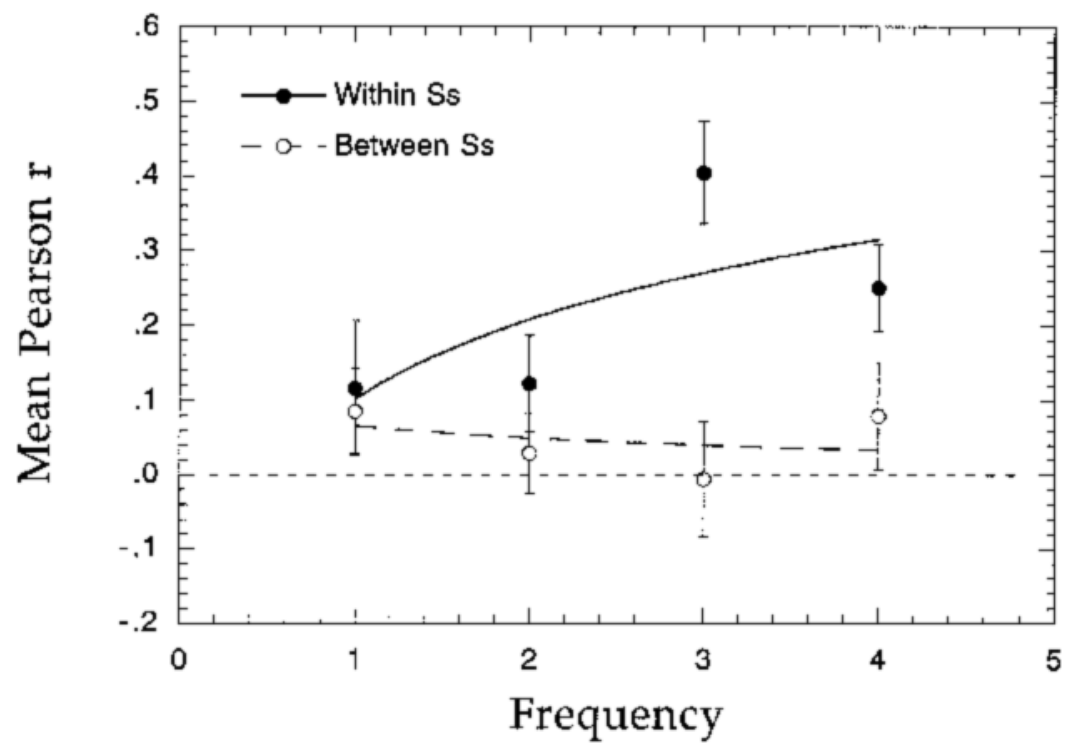

Figure 2. Mean within-subjects and between-subjects correlations with standard errors (Experiment 1). 
60 pairs. For practice, the first 10 test pairs were constructed using filler items from the beginning and end of the study list. Each of the remaining 50 test pairs was made up of words of the same study frequency. At each frequency $(0-4), 5$ pairs were of related items (A and $\mathrm{A}^{\prime}$ ) and the other 5 were of unrelated items (A and $\mathrm{B}^{\prime}$ ). Each word appeared in only 1 test pair. Two test lists were constructed, counterbalancing the assignment of words to related versus unrelated test pairs. This was done in a way that prevented pair-member swapping (i.e., the pairs $\mathrm{A}+\mathrm{B}^{\prime}$ and $\mathrm{B}+\mathrm{A}^{\prime}$ did not both occur). The 5 study lists crossed with the 2 test lists defined the treatments of the 10 groups.

The test form displayed 60 rows, arranged in three columns. Each row included the pair number $(1-60)$ on the left and the numbers $0-10$ on the right. Subjects were instructed to circle one of the values $0-10$ as a JOSF for each test pair. The computer displayed each test pair in three rows. The top row gave the pair number (1-60). First-half and second-half study words were assigned equally often to the middle and bottom rows. Each such display was on the screen for $8 \mathrm{sec}$, followed by a 1 -sec ISI. Test font was the same as at study. As in Experiment 1, subjects were urged to keep up with the computer and not skip any of the test pairs. A beep sounded during the first $0.5 \mathrm{sec}$ of each ISI to keep subjects on pace.

\section{Results}

The JOSF task of Experiment 2 might have been more demanding than the JOF task of Experiment 1. The required mental operations were, of course, more complex. In addition, the longer rows of numbers may have made it easier to lose one's place on the test form. Six subjects, whose mean JOSFs correlated between .67 and .80 with objective frequency, were judged to have followed instructions inadequately and were dropped from further analysis. Three of these subjects' test forms showed clear evidence of error (double or missed responses). The other 3 subjects had inordinately high mean JOSFs in the zerofrequency condition, again suggesting some misplaced responses. For the remaining subjects, mean JOSFs correlated between .82 and .99 with objective frequency (mean $r=.95$ ). All further analyses were done on the data from these 39 subjects.

Figure 3 presents the mean JOSFs and means of withinsubjects $S D$ s. Qualitatively, the means and $S D$ s displayed a similar pattern: an increase with summed frequency, higher values for related than for unrelated pairs, and a tendency for the related versus unrelated difference to increase with frequency. Orthogonal contrasts on the two measures showed somewhat different patterns, however. In the case of mean JOSF, the linear trend on summed frequency was strong $[t(38)=25.37]$. The main effect of related versus unrelated pairs was not significant $[$ mean $=$ 4.30 vs. $4.13 ; t(38)=1.70, p=.097]$, although it did interact reliably with the linear trend on frequency $[t(38)=$ $3.02, p=.044]$. The assumption discussed earlier that $\mu_{X}$ and $\mu_{Y}$ are unaffected by test-pair similiarity appears approximately correct in spite of the marginal interaction. In the case of $S D$ s, there was a reliable linear trend on frequency $[t(38)=5.44, p<.0001]$ and a main effect of related versus unrelated test pairs [mean $S D=1.58$ vs. $1.38 ; t(38)=3.27, p=.002]$. The interaction was not reliable $[t(38)=1.18, p=.244]$.
It is common for the variability of a dependent measure to increase directly with the mean, and this raises the question of whether the related versus unrelated difference in $S D$ s - the effect of primary theoretical interest here-could be an artifact of the related versus unrelated difference in means. This seems unlikely, and not just because the latter difference failed to reach statistical significance. Variability typically increases with the mean when the dependent measure has a floor but is not approaching a ceiling. The most familiar example is reaction time. Frequency judgments are often made on an open-ended scale lacking a ceiling, but in this experiment they were limited to the range $0-10$. As judgments increase, the upper limit constrains the mean and especially constrains the variability (which must be 0 when the mean hits the top of the scale). In this experiment, mathematical constraints on $S D$ s should be weakest in the middle of the scale, around JOSF $=5$, and increase as the mean approaches one of the endpoints. This does not fit the pattern evident in Figure 3. At a summed frequency of 8, the related-pair mean is closer to maximum than is the unrelated-pair mean, but the related-pair $S D$ is higher. Interpretation of a difference in $S D$ s can be complicated by a difference in means, but in this case the difference in means cannot explain the difference in $S D$ s.

It has been known for some time that experimental subjects can make quite accurate JOFs, even in the absence of any forewarning (Hasher \& Chromiak, 1977; Hintzman, 1969; Howell, 1973). The ability to make accurate JOSFs does not seem to have been demonstrated previously, however. In view of the high level of accuracy, it is of interest to compare JOSF performance in Experiment 2 with JOF performance in Experiment 1. A simple hypothesis is that the subjects in Experiment 2 made a JOF for either test word covertly, exactly as the Experiment 1 subjects did overtly, and arrived at a JOSF by summing the two values. This is at least a mathematical possibility, because the minimum and maximum of the JOSF scale ( 0 and 10$)$ were double the minimum and maximum of the JOF scale (0 and 5). There are, of course, several reasons why this hypothesis might not hold. These include the extra computational demands of the JOSF task and the consequent difficulty in equating time constraints across the two experiments. Nevertheless, the hypothesis provides a baseline against which JOSF performance can be evaluated.

The most straightforward way to derive JOSF predictions from the JOF data is to sum individual JOFs from Experiment 1 for related and unrelated word pairs, exactly as these were defined in the test phase of Experiment 2. The scattergrams in Figure 4 show how well the means and $S D$ s of JOF sums (Experiment 1) predict the means and $S D$ s of JOSFs (Experiment 2). In the case of means, $99.2 \%$ of the variance was accounted for by a regression line with a slope of 1.03 and an intercept of 0.38 . In the case of $S D \mathrm{~s}, 91 \%$ of the variance was accounted for by a line with a slope of 0.77 and an intercept of 0.46 . In gen- 


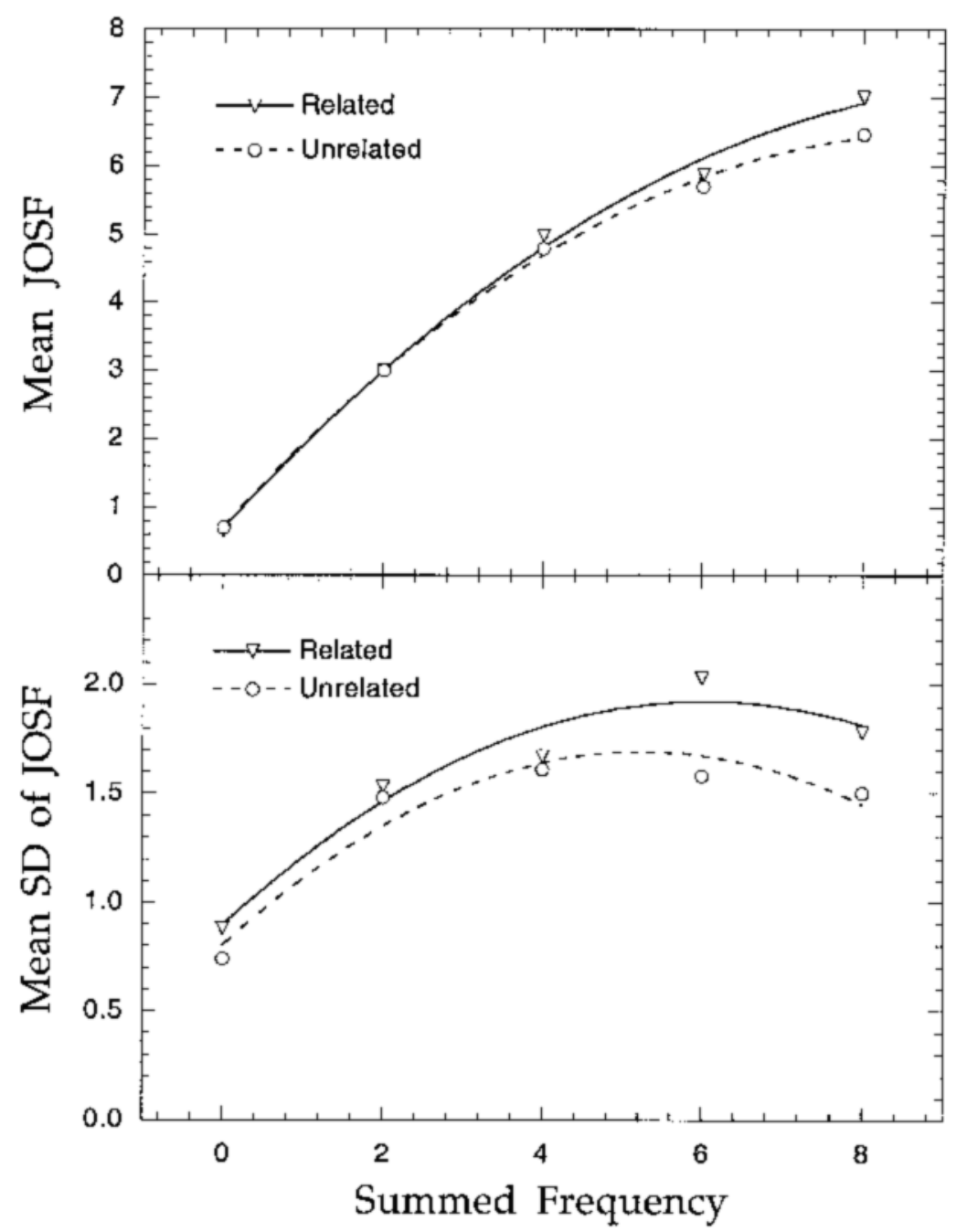

Figure 3. Mean data on judgments of summed frequency (JOSF) for related and unrelated word pairs in Experiment 2.

eral, although JOSFs were somewhat noisier than predicted on the basis of JOFs, the predictions were surprisingly accurate. ${ }^{3}$

\section{GENERAL DISCUSSION}

According to global matching models, the test-pair similarity effect in forced-choice recognition occurs because similar test items produce summed activation levels that are correlated. The Minerva 2 model (Hintzman, 1988) assumes that apparent frequency, as well as recognition memory, is based on global matching. If this is correct, evidence of such correlations should also appear in frequencyjudgment tasks. Experiment 1 confirmed this hypothesis directly, by showing that JOFs for semantically related words are positively correlated. Experiment 2 confirmed it indirectly, by showing that JOSFs are more variable when test pairs are related than when they are unrelated.

It is worth considering other ways in which one might explain the outcome of Experiment 1. One possibility is that the subjects somehow discovered that the frequencies of related words were equal. Accordingly, when they encountered $\mathrm{A}^{\prime}$ in the second half of the test list, they tried to recall and reproduce the JOF that they earlier gave to A. There are two versions of this hypothesis. One is that the subjects discovered the equal-frequency rule during the study list. This version is problematic because the subjects were unlikely to have anticipated that the experiment would require them to remember frequencies, and because-from the subject's point of view-an item's presentation frequency was not established until the study list had ended. At best, each word might be thought of as 
having a running count, which may be incremented at any time during study. During most of the study list, the running counts of related words were not equal.

The JOSF data of Experiment 2, which had study conditions identical to Experiment 1, provide an empirical test of the foregoing hypothesis. Subjects who believe that the frequencies of related test pairs are usually the same should be more inclined to give even-valued JOSFs to related-word pairs than to unrelated-word pairs. The proportions of even-valued JOSFs in Experiment 2 were in fact virtually identical for the two kinds of pairs$59.6 \%$ for related pairs, and $59.4 \%$ for unrelated pairs. (The correct JOSFs, of course, were always even values.) These results offer no support for the idea that the subjects discovered during study that the frequencies of related words were equal.

The second version of the hypothesis is that the subjects formulated the equal-frequency rule during testing. Suppose that when a certain word $\mathrm{A}^{\prime}$ occurs on the test, three things happen: the subject generates a JOF to $A^{\prime}$; the subject is reminded of $A$ and of the JOF that was given to $A$ earlier; and the subject notices that the two JOFs are the same. This suggests the general rule that related words have the same frequency - a rule that is confirmed by and influences subsequent JOFs. Here, discovery of the equalfrequency rule is assumed to depend on the power of the test item $\mathrm{A}^{\prime}$ to remind the subject of $\mathrm{A}$, even after an interval of several minutes. Unlike the hypothesis that the equal-frequency rule is discovered during study, this hypothesis does not predict different percentages of evenvalued JOSFs for related pairs than for unrelated pairs in the second experiment, because long-term retrieval of $\mathrm{A}$ by $\mathrm{A}^{\prime}$ would not be a factor when the pair-testing procedure is used.

Another possibility is that correlated JOFs of Experiment 1 derive not from conscious discovery of a rule, but from automatic effects of implicit memory. When the subject makes a numerical JOF to word A, an association is formed between $\mathrm{A}$ and the number. Later in the test list, $\mathrm{A}^{\prime}$ reminds the subject of $\mathrm{A}$, which automatically activates the association. This automatic reminding in turn intrudes into the formulation of the new JOF, to a degree sufficient to induce the observed correlations.

The empirical evidence regarding such associated intrusions in JOFs is generally negative. Hintzman, Nozawa, and Irmscher (1982) had subjects learn picture-digit pairs and then had the subjects give JOFs to the pictures. There was no evidence that the JOFs were influenced by the associated digits. Similarly, Hintzman (1982) had subjects memorize the spatial numerosities of words on slides and later judge how many slides had displayed each test word (the JOF task). There was no evidence that JOFs were influenced by the spatial numerosities. In a related study, Jonides and Jones (1992) found that JOFs to quantitative words (e.g. twins, quadrangle, couple, triplets) were less accurate than JOFs to control words, a result they attributed to direct coding of frequency of occurrence. Their control words, however, appear not to have been matched with the quantitative words on semantic similarity. High similiarity in itself could account for the reported difference in accuracy of JOFs. Crucially, and consistent with the earlier results, Jonides and Jones found no evidence for the intrusion of pre-experimental number associates into the JOFs of quantitative words (e.g., $\mathrm{JOF}=2$, for $t$ wins). Although these negative outcomes are not definitive, they tend to undermine the hypothesis that the correlations observed in Experiment 1 were caused by automatic associative intrusions.

Experiment 1 provides direct evidence that JOFs for related words are correlated. However, as we have just seen, either deliberate or automatic use of earlier JOFs might explain the same outcome. Support for the globalmatching account of the obtained correlations, therefore, needs to be reinforced by less direct evidence. Such evidence is provided by Experiment 2 .

The strongest argument for the global-matching account of the JOF correlations rests on its ability to integrate the finding with others that, on the surface, may appear to be unrelated. According to the Minerva 2 model, JOFs of related words are correlated because they produce summed activation levels that are correlated. Such correlations also figure into Equation 1 when memory judgments are based on activation differences and into Equation 2 when judgments are based on activation sums. Equation 1 is manifested by the test-pair similarity effect in forced-choice recognition (Clark \& Gronlund, 1996; Hall, 1979; Hintzman, 1988; Tulving, 1981), and Equation 2 is manifested in the JOSF variability difference demonstrated by Experiment 2 .

By forging this link between recognition memory and frequency judgments, the present results add to the list of evidence supporting the proposition that the same basic processes underlie performance in both of these tasks. Other evidence for a close relationship between recognition and JOFs includes the following: First, at a conceptual level, the old versus new recognition choice (frequency $=$ 0 vs. frequency $>0$ ) can be seen as differing only quantitatively from choices among frequencies of $1,2,3$, and so on. Second, the Minerva 2 model, which explicitly describes a small set of processes common to recognition and frequency decisions, simulates recognition and JOF results over a range of presentation frequencies that includes frequency $=0$ (Hintzman, 1988). Third, forced-choice frequency-discrimination performance on pairs that include frequency $=0$ appears to be commensurate with performance on pairs that exclude frequency $=0$. For example, the accuracy of the 2 versus 1 frequency discrimination can be predicted from the joint accuracy of the 2 versus 0 and 1 versus 0 discriminations (Hintzman, Curran, \& Caulton, 1995). Fourth, 24-h forgetting functions for frequency discriminations that exclude frequency $=0$ ( 2 vs. 1,4 vs. 2 , and 4 vs. 1$)$ appear parallel to the forgetting function for frequency 1 versus 0 (Hintzman \& Stern, 1984). Fifth, evidence on retrieval dynamics suggests that the ability to discriminate frequencies of 2 versus 1 begins as early in the retrieval episode as 

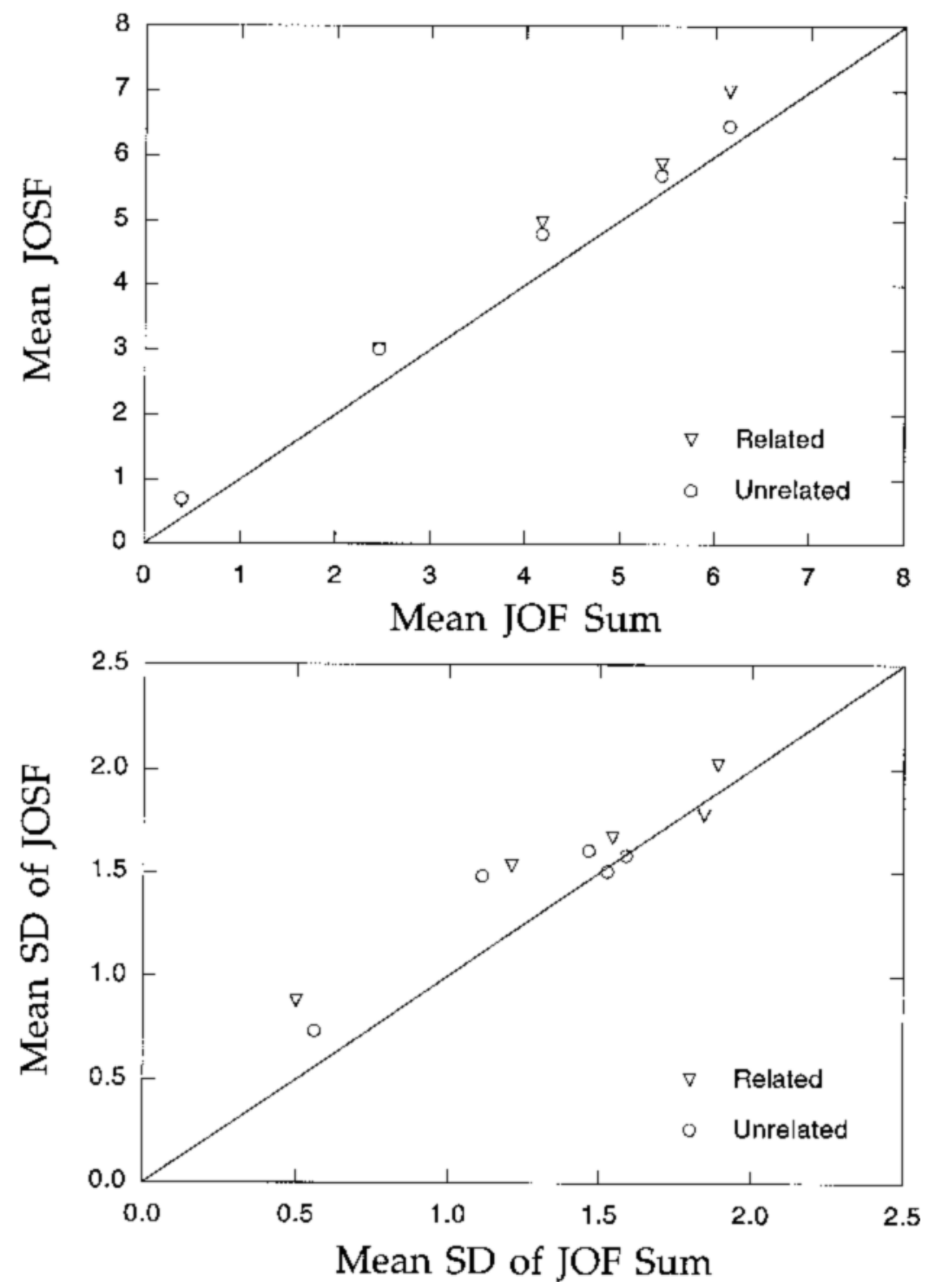

Figure 4. Prediction of Experiment 2 data (ordinate) from Experiment 1 data (abscissa). JOSF $=$ judgment of summed frequency; JOF $=$ judgment of frequency.

does the ability to make recognition decisions ( 1 vs. 0 and 2 vs. 0 ). The ability to judge other details of a word's prior presentation begins about $100 \mathrm{msec}$ later (Gronlund, Edwards, \& Ohrt, 1997; Hintzman, 2000; Hintzman \& Caulton, 1997; Hintzman \& Curran, 1994). Sixth, encoding manipulations such as levels of processing (Fisk \& Schneider, 1984; Naveh-Benjamin \& Jonides, 1985; Rose \& Rowe, 1976; Rowe, 1974), read versus generate (Greene, 1988), and spacing of repetitions (Hintzman, 1969; Hintzman \& Block, 1970; Hintzman \& Rogers, 1973; Underwood, 1969) have effects on JOF magnitude that parallel their effects on recognition accuracy. Seventh, materials manipulations that produce mirror effects in recognition memory (common vs. rare words, and nouns vs. consonant strings) also produce mirror effects in the discrimination of frequencies (Greene \& Thapar, 1994). Taken as a whole, this evidence suggests that recognition judgments and frequency judgments have a common underlying mechanism (for some contrary evidence, see Proctor, 1977).

Recent testing of memory models has depended heavily on data from the recognition-memory task (Clark \& Gronlund, 1996) and, with the exception of the Minerva 2 model (Hintzman, 1988), has virtually ignored memory for frequency. Gillund and Shiffrin (1984, p. 48) suggested that global-matching strength might provide a basis for frequency discrimination in the SAM model. Although this idea was not implemented, it appears generally consistent with the approach developed with Minerva 2. A later version of SAM (Shiffrin, Ratcliff, \& 
Clark, 1990) includes a differentiation process, by which repetition of an item decreases the tendency for other items to activate the repeated item's memory representation. If such differentiation were to completely eliminate the tendency for $\mathrm{A}^{\prime}$ to activate the trace of $\mathrm{A}$, and vice versa, this could eliminate the global-matching correlations that underlie the present predictions for JOF. In the applications of the model discussed by Shiffrin et al. (1990), however, differentiation is never complete. Moreover, the focus is on differentiation of A from an unrelated item, not from a related item such as $\mathrm{A}^{\prime}$. Differentiation of A from $\mathrm{A}^{\prime}$ could asymptote at a level that still permitted substantial mutual cross activation. Thus, by adopting the right parameter values, a version of SAM adapted to the JOF task might accommodate the present results.

In more recent developments, however, SAM has been superseded by REM (Shiffrin \& Steyvers, 1997). In REM, repetition has essentially one function, which is to increase the precision of the memory representation of the repeated item. At retrieval, features of the test item are sampled, and the stored representations are used to calculate the log odds that those features came from each old item, as opposed to a typical new item, in a recognitiondecision that can be characterized as Bayesian. A recognitionmemory model proposed by McClelland and Chappell (1998) makes slightly different assumptions, but they lead to nearly identical equations.

These new models have a number of admirable properties. Foremost among them is the ability to produce recognition mirror effects, which are problematic for globalmatching models such as SAM and Minerva 2. However, there seems to be no obvious way to extend the Bayesian decision process to frequency judgments. Indeed, Shiffrin and Steyvers (1997, footnote 5) suggest that JOFs would require another sort of mechanism. And although McClelland and Chappell (1998, p. 730) do deal with data from the frequency-judgment task, they focus on the recognition decision, JOF $=0$ versus JOF $>0$, and explicitly exclude from consideration the tendency for JOFs of 1,2 , 3 , and so on to track increases in objective frequency.

Indirectly, the development of these new models puts the spotlight on memory for frequency. If the processes that underlie recognition and JOFs are essentially the same, these and other recognition-memory models will have to address data from both kinds of tasks. If the processes are fundamentally different, it should be possible to demonstrate this, to characterize the difference analytically, and to explain the commonalities that have appeared in the literature, including the correlational effects demonstrated in the present experiments.

\section{REFERENCES}

Clark, S. E., \& Gronlund, S. D. (1996). Global matching models of recognition memory: How the models match the data. Psychonomic Bulletin \& Review, 3, 37-60.

Clark, S. E., Hori, A., \& Callan, D. E. (1993). Forced-choice associative recognition:Implications for global-memory models. Journal of Experimental Psychology: Learning, Memory, \& Cognition, 19, 871-881.

EICH, J. M. (1982). A composite holographic associative recall model. Psychological Review, 89, 627-661.

FISK, A. D., \& SchNeIDER, W. (1984). Memory as a function of attention, level of processing, and automatization. Journal of Experimental Psychology: Learning, Memory, \& Cognition, 10, 181-197.

Gillund, G., \& Shiffrin, R. M. (1984). A retrieval model for both recognition and recall. Psychological Review, 91, 1-67.

Greene, R. L. (1988). Generation effects in frequency judgment. Journal of Experimental Psychology: Learning, Memory, \& Cognition, 14, 298-304.

Greene, R. L., \& Thapar, A. (1994). Mirror effect in frequency discrimination. Journal of Experimental Psychology: Learning, Memory, \& Cognition, 20, 946-952.

Gronlund, S. D., Edwards, M. B., \& OhrT, D. D. (1997). Comparison of the retrieval of item versus spatial position information. Journal of Experimental Psychology: Learning, Memory, \& Cognition, 23, 1261-1274.

Hall, J. F. (1979). Recognition as a function of word frequency. American Journal of Psychology, 92, 497-505.

Hasher, L., \& Chromiak, W. (1977). The processing of frequency information: An automatic mechanism? Journal of Verbal Learning \& Verbal Behavior, 16, 173-184.

Hintzman, D. L. (1969). Apparent frequency as a function of frequency and the spacing of repetitions. Journal of Experimental Psychology, 80, 139-145.

Hintzman, D. L. (1982). Are presentation frequency and spatial numerosity distinct attributes of memory? Bulletin of the Psychonomic Society, 20, 196-198.

Hintzman, D. L. (1988). Judgments of frequency and recognition memory in a multiple-trace memory model. Psychological Review, 95, 528-551.

Hintzman, D. L. (2000). Memory judgments. In E. Tulving \& F. I. M. Craik (Eds.), The Oxford handbook of memory (pp. 165-195). Oxford: Oxford University Press.

Hintzman, D. L., \& Block, R. A. (1970). Memory judgments and the effects of spacing. Journal of Verbal Learning \& Verbal Behavior, 9 , 561-566.

Hintzman, D. L., \& Caulton, D. A. (1997). Recognition memory and modality judgments: A comparison of retrieval dynamics. Journal of Memory \& Language, 37, 1-23.

Hintzman, D. L., \& CuRRan, T. (1994). Retrieval dynamics of recognition and frequency judgments: Evidence for separate processes of familiarity and recall. Journal of Memory \& Language, 33, 1-18.

Hintzman, D. L., Curran, T., \& Caulton, D. A. (1995). Scaling the episodic familiarities of pictures and words. Psychological Science, 6, 308-313.

Hintzman, D. L., Nozawa, G., \& Irmscher, M. (1982). Frequency as a nonpropositional attribute of memory. Journal of Verbal Learning \& Verbal Behavior, 21, 127-141.

Hintzman, D. L., \& Rogers, M. K. (1973). Spacing effects in picture memory. Memory \& Cognition, 1, 430-434.

Hintzman, D. L., \& Stern, L. D. (1984). A comparison of forgetting rates in frequency discrimination and recognition. Bulletin of the Psychonomic Society, 22, 409-412.

Howell, W. C. (1973). Representation of frequency in memory. Psychological Bulletin, 80, 44-53.

Humphreys, M. S., Bain, J. D., \& Pike, R. (1989). Different ways to cue a coherent memory system: A theory for episodic, semantic, and procedural tasks. Psychological Review, 96, 208-233.

JoNidEs, J., \& Jones, C. M. (1992). Direct coding for frequency of occurrence. Journal of Experimental Psychology: Learning, Memory, \& Cognition, 18, 368-378.

LeICHT, K. L. (1968). Recall and judged frequency of implicitly occurring words. Journal of Verbal Learning \& Verbal Behavior, 7, 918-923.

McClelland, J. L., \& Chappell, M. (1998). Familiarity breeds differentiation: A subjective likelihood approach to the effects of experience in recognition memory. Psychological Review, 105, 724-760.

Murdock, B. B., JR. (1982). A theory for the storage and retrieval of item and associative information. Psychological Review, 89, 609-626. 
MuRDOCK, B. B., JR. (1999). Item and associative interactions in shortterm memory: Multiple memory systems? International Journal of Psychology, 34, 427-433.

Naveh-Benjamin, M., \& Jonides, J. (1985). The effects of rehearsal on frequency coding. Bulletin of the Psychonomic Society, 23, 387-390.

Proctor, R. W. (1977). The relationship of frequency judgments to recognition: Facilitation of recognition and comparison to recognitionconfidence judgments. Journal of Experimental Psychology: Human Learning \& Memory, 3, 679-689.

RAtCliff, R. \& McKoon, G. (1988). A retrieval theory of priming in memory. Psychological Review, 95, 385-408.

Rose, R. J., \& Rowe, E. J. (1976). Effects of orienting task and spacing of repetitions on frequency judgments. Journal of Experimental Psychology, 2, 142-152.

Rosenthal, R. \& Rosnow, R. L. (1985). Contrastanalysis. Cambridge: Cambridge University Press.

RowE, E. J. (1974). Depth of processing in a frequency judgment task. Journal of Verbal Learning \& Verbal Behavior, 13, 638-643.

Shiffrin, R. M., RATClifF, R, \& Clark, S. (1990). The list-strength effect. II. Theoretical mechanisms. Journal of Experimental Psychology: Learning, Memory, \& Cognition, 16, 179-195.

Shiffrin, R. M., \& Steyvers, M. (1997). A model for recognition memory: REM-retrieving effectively from memory. Psychonomic Bulletin \& Review, 4, 145-166.

Thurstone, L. L. (1927). A law of comparative judgment. Psychological Review, 34, 273-286.

Tulving, E. (1981). Similarity relations in recognition. Journal of Verbal Learning \& Verbal Behavior, 20, 479-496.
UNDERWoOd, B. J. (1969). Some correlates of item repetition in freerecall learning. Journal of Verbal Learning \& Verbal Behavior, 8 , 83-94.

\section{NOTES}

1. The present predictions regarding similarity effects hold also when frequencies are unequal. An orthogonal manipulation of first-half and second-half frequencies $0-4$, however, would entail 25 different conditions. The five equal-frequency cells were chosen for practical reasons.

2. Strictly speaking, within-subjects correlations could also be influenced by subject-item interactions of nonepisodic origin. Regardless of actual frequency, for example, one subject might be predisposed to give high JOFs to kinds of nuts and another to give high JOFs to legal professions. To the extent that such predispositions exist, however, one would expect many or most to be shared across subjects. Therefore, nonepisodic subject-item interactions are a likely source of within-subjects covariance only to the extent that nonepisodic item effects are demonstrated.

3. A reviewer suggested that the prediction of Equation 2 could be also tested by asking whether the JOF sums for related pairs are greater than those for unrelated pairs within the data of Experiment 1. Given the computed correlations, however, this "prediction" has to be true, because the standard algorithm for computing the Pearson correlation is algebraically equivalent to Equation 2.

(Manuscript received August 8, 2000; revision accepted for publication February 9, 2001.) 